\title{
ESTUDO EM ESCALA GENÔMICA DA Clostridium acetobutylicum ATCC 824 PARA A BIOPRODUÇÃO DE HIDROGÊNIO E CRIAÇÃO DE UM MODELO REGULATÓRIO
}

\section{E. MENDES ${ }^{1}$ e A. FURIGO $\mathrm{Jr}^{2}$}

${ }^{1}$ Universidade do Extremo Sul Catarinense, Departamento de Engenharia Química

${ }^{2}$ Universidade Federal de Santa Catarina, Departamento de Engenharia Química e Engenharia de Alimentos

E-mail para contato: erlon@unesc.net

RESUMO - Neste trabalho foi feito um modelo com regulação da ClostridiumacetobutylicumATCC 824, tendo como base o modelo não regulado mais recente, sendo seus resultados devidamente validados com dados experimentais para diferentes substratos, reproduzidos em Análise de Balanço de Fluxos com regulação (RFBA) utilizando o COBRA Toolbox, tanto em estado pseudo-estacionário quanto dinâmico.

A análise de sensibilidade deste modelo evidenciou grande sensibilidade específica para a produção de hidrogênio utilizando substratos como sacarose, maltose e lactose em substituição a glicose. Os testes experimentais revelaram taxas idênticas de produção de hidrogênio e biomassa a partir de glicose, sacarose e maltose, mas inferiores para lactose.

A análise de deleção de genes revelou a deleção de uma das reações do metabolismo do butanoato, codificada pelo gene CAC1742, como a melhor alternativa para maximizar a produção de hidrogênio por C. acetobutylicum.

\section{INTRODUÇÃO}

A engenharia metabólica é uma grande área de estudos visando utilizar informações do metabolismo celular a nível genômico, técnicas de balanço de massa e rotinas computacionais avançadas para obter melhores rendimentos na produção de um metabólito de interesse por um microrganismo específico. Estes estudos geralmente indicam as rotas mais eficientes de mutação do microrganismo alvo.

O Grupo de Pesquisa de Sistemas Biológicos da Universidade da Califórnia integraram uma série de funções envolvendo principalmente estudos de engenharia metabólica, o COBRA Toolbox(BECKER et al., 2007; SCHELLENBERGER et al., 2011). Deste toolbox, as funções relativas a Análise de Balanço de Fluxo (FBA, do inglês Flux Balance Analysis) foram as mais utilizadas neste trabalho. Esta análise utiliza técnicas de otimização linear para predizer todos os fluxos de um organismo reconstruído in silico, submetidos a restrições e a uma função objetivo, que geralmente é a maximização do crescimento celular.

Um organismo recentemente reconstruído in silico foi a Clostridium acetobutylicum ATCC 824, conhecida por produzir grandes quantidades de acetato, butirato e hidrogênio (SALIMI, ZHUANG e MAHADEVAN, 2010), sendo a produção de hidrogênio o objetivo do estudo de engenharia metabólica deste trabalho. 
Uma análise útil aos estudos deste trabalho é a análise de sensibilidade. A análise de sensibilidade, desenvolvida por Stephanopoulos, Aristidou e Nielsen (1998), gera resultados que indicam quais fluxos extracelulares causam maior impacto sobre um fluxo escolhido.

Juntamente com a reconstrução metabólica, os modelos in silico mais atuais contém também uma representação booleana de parte da rede regulatória transcricional. Em resposta a estímulos externos e internos, fatores transcricionais ativam ou reprimem genes associados a reações metabólicas. Esta regulação melhora a fidelidade preditiva do modelo metabólico pela imposição de um contexto adicional à restrições de genes específicos, podendo tanto induzi-los completamente quanto reprimi-lostotalmente.

\section{MATERIAIS E MÉTODOS}

\subsection{A construção do modelo com regulação para a $C$. acetobutylicum ATCC 824}

O modelo de Salimi, Zhuang eMahadevan(2010) foi escolhido para a inserção de regras de regulação.

As regras de regulação adicionadas ao modelo tiveram como base as evidências experimentais de Vasconcelos, Girbal eSoucaille(1994), relativo à regulação por glicerol, Tangney e Mitchell (2000), relativo à regulação por sacarose, Tangney, Winters e Mitchell (2001), relativo à regulação por maltose, e finalmente Yuet al.(2007), relativo à regulação por lactose.

Segundo Vasconcelos, Girbal eSoucaille(1994), o glicerol inibe a enzima NADHferredoxinaoxiredutase.

Em Tangney e Mitchell (2000), a atividade do sistema fosfotransferase(Phosphotransferase System - PTS) dependente do fosfoenolpiruvato e específico para a sacarose é inibida quando glicose está presente. Tangney, Winters e Mitchell (2001) afirmam que o sistema PTS específico para a maltose também é inibido pela presença de glicose. Segundo Yuet al.(2007), o mesmo tipo de inibição também ocorre para a lactose.

Com estas informações regulatórias, foram criadas as regras de regulação que fizeram parte de um novo modelo.

Em Tangney e Mitchell (2000); Tangney, Winters e Mitchell (2001); Yuet al.(2007), os dados experimentais foram apresentados em um sistema dinâmico e a validação do modelo foi feita com a função dynamicRFBA do COBRA Toolbox. Já a validação com os dados de Vasconcelos, Girbal eSoucaille(1994) foi feita utilizando a função optimizeRegModel, que faz a FBA em um sistema contínuo.

\subsection{Análise de sensibilidade da $C$. acetobutylicum}

A análise de sensibilidade(STEPHANOPOULOS, ARISTIDOU e NIELSEN, 1998) foi feita utilizando a função sensitivity (do autor), executada no modelo com regulação, que tem como base o uso da Equação(1) na matriz estequiométrica $\mathbf{G}$, separada em valores medidos $(\mathrm{m})$ e calculados $(\mathrm{c})$. Neste estudo, foram colocados no 
grupo dos medidos todos os fluxos extracelulares. O símbolo ( $\left(^{\prime}\right)$ indica a trasposta da matriz enquanto (\#) a sua pseudo-inversa.

$$
\frac{d \mathbf{v}_{\mathbf{c}}}{d \mathbf{v}_{\mathbf{m}}}=-\left(\mathbf{G}_{\mathbf{c}}^{\prime}\right)^{\#} \mathbf{G}_{\mathbf{m}}^{\prime}
$$

\subsection{Nocaute de genes da $C$. acetobutylicum}

O estudo do nocaute de genes para possíveis mutações que aumentariam a produção de hidrogênio foi feito em um modelo reproduzido de Lee et al.(2008)no COBRA Toolbox a partir das funções OptKnock e GDLS.

\subsection{Procedimento experimental para o crescimento da $C$. acetobutylicum com diferentes substratos}

O crescimento consiste inicialmente de uma reativação em um meio clostridial reforçado (ReinforcedClostridialMedium - RCM) (Tabela 1) previamente autoclavado a $121{ }^{\circ} \mathrm{C} / 15 \mathrm{~min}$. A reativação deve ser feita durante 24 horas em jarro de anaerobiose a $37{ }^{\circ} \mathrm{C}$ e pH 6,8, aqui chamada de pré-cultura. A transferência das bactérias para o meio RCM é feita em capela de fluxo laminar previamente esterilizada com álcool $70 \%$ e luz UV. Depois de inoculado, o meio deve ser colocado no jarro de anaerobiose juntamente com um sachet do produto Anaerogen da Oxoid, que garantirá a anaerobiose.

\begin{tabular}{ll} 
Tabela 1- Meio clostridial reforçado $(\mathrm{RCM})$ para a pré-cultura. \\
\hline Composto & $\begin{array}{l}\text { Concentração } \\
(\mathrm{g} / \mathrm{L})\end{array}$ \\
\hline Glicose-D & 5,0 \\
Peptona & 10,0 \\
Cloreto de Sódio & 5,0 \\
Amido Solúvel & 1,0 \\
Extrato de Levedura & 3,0 \\
Extrato de Carne & 10,0 \\
Cloridrato de Cisteína (1-hidr) & 0,5 \\
Acetato de Sódio & 3,0 \\
\hline
\end{tabular}

Fonte: Fundação André Tosello

Um novo meio (Tabela 2), agora destinado a fermentação propriamente dita, é então inoculado a $10 \%$ v/v utilizando o meio RCM com as bactérias reativadas.

Tabela 2 - Meio de fermentação.

\begin{tabular}{ll}
\hline Composto & $\begin{array}{l}\text { Concentração } \\
(\mathrm{g} / \mathrm{L})\end{array}$ \\
\hline Glicose-D & 20,0 \\
Peptona & 4,0 \\
Cloreto de Sódio & 3,0 \\
Extrato de Levedura & 1,0 \\
Extrato de Carne & 1,0 \\
Cloridrato de Cisteína (1-hidr) & 0,5 \\
Acetato de Sódio & 0,1 \\
\hline
\end{tabular}


Fonte: Fundação André Tosello

Após a inoculação, os frascos foram tampados com ronhas de silicone contendo dois tubos de vidro seguidos de mangueiras de silicone para entrada e saída de gases. Borbulhou-se nitrogênio para remover o oxigênio do interior do frasco durante 10 minutos. Para todos os frascos, as saídas de gás são acopladas à lavadores de gás contendo solução $1 \mathrm{M}$ de $\mathrm{NaOH}$, para a captura do $\mathrm{CO}_{2}$, e as saídas dos lavadores de gás são finalmente acopladas à provetas invertidas, de onde é quantificado o volume de gás restante produzido (primordialmente hidrogênio). A fermentação é feita a $37^{\circ} \mathrm{C} \mathrm{em}$ um agitador orbital a 120 rpm conforme mostrado na Figura 1.

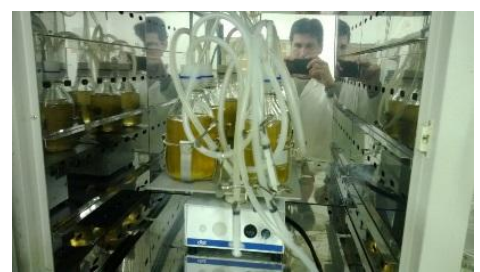

(a)

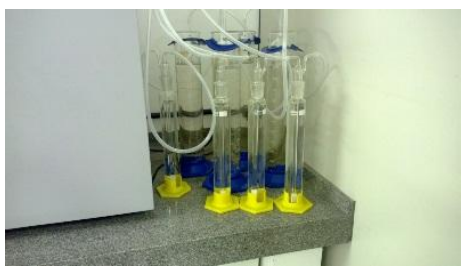

(b)

Figura 1 - Aparato experimental utilizado.

Fonte: Do autor.

O volume do gás foi determinado por proveta invertida. A concentração de células foi determinada por turbidimetria a $570 \mathrm{~nm}$ e por peso seco. A concentração de glicose, e outros açúcares nas variações de substrato, foi determinada pelo método ácido antrona-sulfúrico (DUBOIS et al., 1956). O pH do meio também foi monitorado.

Com este procedimento, investigou-se a resposta na produção de hidrogênio quanto a utilização de outros substratos, sugeridos durante a análise de sensibilidade.

\section{RESULTADOS}

\subsection{Análise de sensibilidade da $C$. acetobutylicum}

A análise de sensibilidade mostrou grande influência dos fluxos relativos ao consumo de sacarose, lactose e maltose, respectivamente, apresentaram alta sensibilidade, sendo provavelmente opções melhores do que a glicose como substrato para a produção de hidrogênio, o que foi experimentalmente investigado e os resultados mostrados na seção 3.3 .

\subsection{Nocaute de Genes da C. acetobutylicum}

As funções OptKnock e GDLS apresentaramo nocaute da reação BUTAN3 (Acetil-CoA $+\mathrm{HPO}_{4}$ Acetil fosfato + CoA), com gene de LocusNumber CAC1742, como a melhor opção para maximizar a produção de hidrogênio.

Esta hipótese ainda precisa ser testada experimentalmente.

\subsection{O crescimento da $C$. acetobutylicum ATCC824 em sacarose, maltose e lactose vs. glicose}


A partir da análise das concentrações de células $(\mathrm{X})$, substrato $(\mathrm{S})$ e produto $(\mathrm{P})$, fez-se o estudo cinético para os diferentes substratos tendo a equação de Monod, Equação (2), como equação cinética.

$$
\mu=\mu_{m} \frac{S}{K_{S}+S}
$$

Para o estudo da sacarose vs. glicose, obteve-se os dados cinéticos e estequiométricos mostrados na Tabela 3.

Tabela 3 - Parâmetros cinéticos e estequiométricos do crescimento em glicose vs. sacarose.

\begin{tabular}{lll}
\hline Parâmetro & Glicose & Sacarose \\
\hline$\mu_{\mathrm{m}}$ & $0,1644 \mathrm{~h}^{-1}$ & $0,1605 \mathrm{~h}^{-1}$ \\
$\mathrm{~K}_{\mathrm{s}}$ & $3 \mathrm{~g}_{\mathrm{S}} / \mathrm{L}$ & $3 \mathrm{~g}_{\mathrm{S}} / \mathrm{L}$ \\
$\mathrm{Y}_{\mathrm{X} / \mathrm{S}}$ & $0,25 \mathrm{~g}_{\mathrm{X}} / \mathrm{g}_{\mathrm{S}}$ & $0,26 \mathrm{~g}_{\mathrm{X}} / \mathrm{g}_{\mathrm{S}}$ \\
$\mathrm{Y}_{\mathrm{X} / \mathrm{P}}$ & $0,37 \mathrm{~g}_{\mathrm{X}} / \mathrm{g}_{\mathrm{P}}$ & $0,39 \mathrm{~g}_{\mathrm{X}} / \mathrm{g}_{\mathrm{P}}$ \\
\hline
\end{tabular}

A simulação do crescimento em batelada, justamente com os dados experimentais da sacarose vs. glicose, são mostrados na Figura 2.

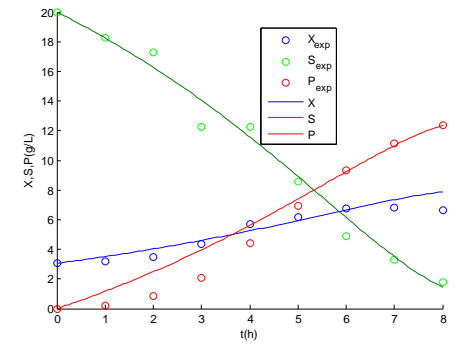

(a)

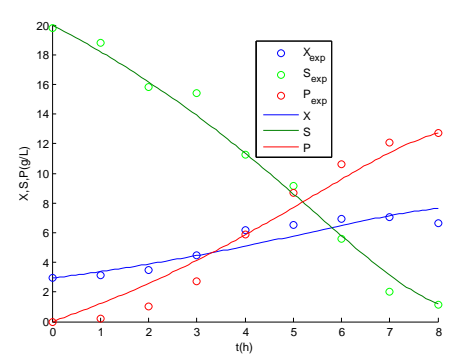

(b)

Figura 2 - Gráfico da simulação em batelada e dados experimentais de X, S e P em função do tempo, sendo (a) o crescimento em glicose e (b) sacarose.

Fonte: Do autor.

A simulação mostra boa concordância com os dados experimentais e os parâmetros cinéticos obtidos evidenciam taxas de crescimento e produção de hidrogênio muito próximas para os dois substratos.

Para o estudo da maltose vs. glicose, obteve-se os dados cinéticos e estequiométricos mostrados na Tabela 4.

Tabela 4 - Parâmetros cinéticos e estequiométricos do crescimento em glicose vs.

maltose.

\begin{tabular}{lll}
\hline Parâmetro & Glicose & Maltose \\
\hline$\mu_{\mathrm{m}}$ & $0,1259 \mathrm{~h}^{-1}$ & $0,1215 \mathrm{~h}^{-1}$ \\
$\mathrm{~K}_{\mathrm{s}}$ & $2 \mathrm{~g}_{\mathrm{S}} / \mathrm{L}$ & $2 \mathrm{~g}_{\mathrm{S}} / \mathrm{L}$ \\
$\mathrm{Y}_{\mathrm{X} / \mathrm{S}}$ & $0,15 \mathrm{~g}_{\mathrm{X}} / \mathrm{g}_{\mathrm{S}}$ & $0,15 \mathrm{~g}_{\mathrm{X}} / \mathrm{g}_{\mathrm{S}}$ \\
$\mathrm{Y}_{\mathrm{X} / \mathrm{P}}$ & $0,22 \mathrm{~g}_{\mathrm{X}} / \mathrm{g}_{\mathrm{P}}$ & $0,25 \mathrm{~g}_{\mathrm{X}} / \mathrm{g}_{\mathrm{P}}$ \\
\hline
\end{tabular}


Assim como para sacarose vs. glicose, a simulação dos dados da maltose vs. glicose também mostrou boa concordância com os dados experimentais (dados não mostrados) e os parâmetros cinéticos obtidos evidenciam taxas de crescimento e produção de hidrogênio muito próximas para os dois substratos.

Para o estudo da lactose vs. glicose, obteve-se os dados cinéticos e estequiométricos mostrados na Tabela 5.

Tabela 5 - Parâmetros cinéticos e estequiométricos do crescimento em glicose vs.

lactose.

\begin{tabular}{lll}
\hline Parâmetro & Glicose & Lactose \\
\hline$\mu_{\mathrm{m}}$ & $0,1280 \mathrm{~h}^{-1}$ & $0,06853 \mathrm{~h}^{-1}$ \\
$\mathrm{~K}_{\mathrm{s}}$ & $2 \mathrm{~g}_{\mathrm{S}} / \mathrm{L}$ & $1 \mathrm{~g}_{\mathrm{S}} / \mathrm{L}$ \\
$\mathrm{Y}_{\mathrm{X} / \mathrm{S}}$ & $0,17 \mathrm{~g}_{\mathrm{X}} / \mathrm{g}_{\mathrm{S}}$ & $0,38 \mathrm{~g}_{\mathrm{X}} / \mathrm{g}_{\mathrm{S}}$ \\
$\mathrm{Y}_{\mathrm{X} / \mathrm{P}}$ & $0,22 \mathrm{~g}_{\mathrm{X}} / \mathrm{g}_{\mathrm{P}}$ & $0,30 \mathrm{~g}_{\mathrm{X}} / \mathrm{g}_{\mathrm{P}}$ \\
\hline
\end{tabular}

A simulação do crescimento em batelada, justamente com os dados experimentais da lactose vs. glicose, são mostrados na Figura 3.

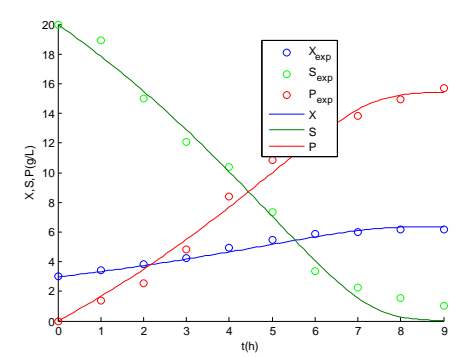

(a)

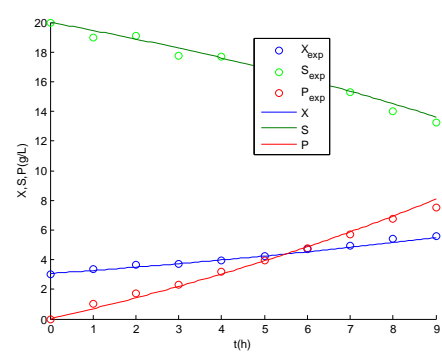

(b)

Figura 3 - Gráfico da simulação em batelada e dados experimentais de X, S e P em função do tempo, sendo (a) o crescimento em glicose e (b) lactose.

Fonte: Do autor.

Diferentemente dos resultados obtidos com sacarose e maltose, as taxas de crescimento e produção de produto foram desfavoráveis para lactose.

Apesar de não terem sido obtidas taxas maiores alimentando-se com sacarose, maltose e lactose, hipótese levantada pela análise de sensibilidade, taxas idênticas foram obtidas com sacarose e maltose, possibilitando substituições por estes substratos devido a fatores econômicos.

\subsection{Simulações do modelo com regulação e validação com dados experimentais}

Após as inserções das regras de regulação e ajustes das restrições, todas as variantes dos modelos com regulação foram validadas com os respectivos dados experimentais. A Figura 4 mostra a validação da regulação da glicose sobre o consumo de sacarose. 


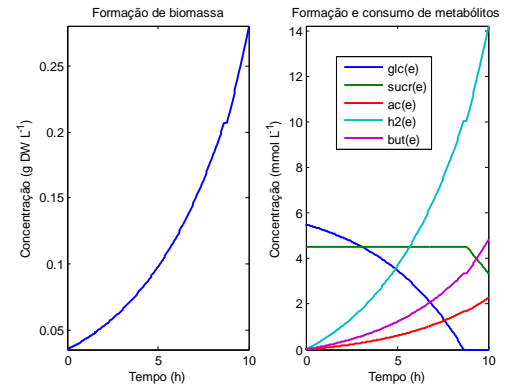

(a)

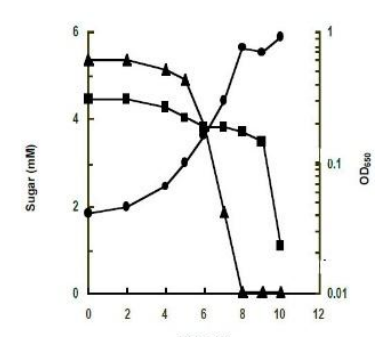

(b)

Figura 4 - Gráfico de regulação da glicose sobre o consumo de sacarose. Dados simulados (a) vs. dados experimentais (b), onde $\boldsymbol{\Delta}$ representa a concentração de glicose, $\mathbf{\square}$ a concentração de sacarose e $\bullet$ a concentração de biomassa.

Fonte: (a) Simulação do autor e (b) Tangney e Mitchell (2000).

A Figura 4valida a simulação dinâmica com regulação, mostrando resultados muito próximos dos experimentais. Validações semelhantes também foram feitas com a regulação da maltose e lactose (dados não mostrados).

Devido à natureza dos dados experimentais de Vasconcelos, Girbal eSoucaille(1994), a validação do modelo para a regulação por glicerol foi feita via FBA com regulação para sistemas contínuos e também apresentou boa concordância com os dados experimentais (dados não mostrados).

\section{CONCLUSÃO}

Para utilizar as funções de regulação gênica do COBRA Toolbox, um modelo com regulação para a $C$. acetobutylicum ATCC 824 foi criado e validado com dados experimentais.

A análise de nocaute de genes apresentouo nocaute da reação de LocusNumber CAC1742, como a melhor opção para maximizar a produção de hidrogênio, hipótese ainda não validada experimentalmente.

A análise de sensibilidade levantou a hipótese de substituição da glicose por sacarose, maltose ou lactose como boas alternativas para maximizar a produção de hidrogênio. Testando esta hipótese experimentalmente, observou-se que a glicose pode mesmo ser substituída por sacarose e maltose, mas não por lactose, sem perder o rendimento para células e hidrogênio.

\section{REFERÊNCIAS}

BECKER, S. A. et al. Quantitative prediction of cellular metabolism with constraintbased models: the COBRA Toolbox. Nat Protoc, v. 2, n. 3, p. 727-38, 2007. ISSN 1750-2799 (Electronic)

1750-2799

(Linking).

Disponível

em:

<http://www.ncbi.nlm.nih.gov/pubmed/17406635>. 
DUBOIS, M. et al. Colorimetric Method for Determination of Sugars and Related Substances. Analytical Chemistry, v. 28, n. 3, p. 350-356, 1956/03/01 1956. ISSN 0003-2700. Disponível em: <http://dx.doi.org/10.1021/ac60111a017>. Acesso em: 2012/05/08.

LEE, J. et al. Genome-scale reconstruction and in silico analysis of the Clostridium acetobutylicum ATCC 824 metabolic network. Appl Microbiol Biotechnol, v. 80, n. 5, p. 849-62, Oct 2008. ISSN 1432-0614 (Electronic)
$0175-7598$
(Linking).
Disponível
em:

<http://www.ncbi.nlm.nih.gov/pubmed/18758767>.

SALIMI, F.; ZHUANG, K.; MAHADEVAN, R. Genome-scale metabolic modeling of a clostridial co-culture for consolidated bioprocessing. Biotechnology Journal, v. 5, n. 7, p. 726-738, 2010. ISSN 1860-7314. Disponível em: <http://dx.doi.org/10.1002/biot.201000159>.

SCHELLENBERGER, J. et al. Quantitative prediction of cellular metabolism with constraint-based models: the COBRA Toolbox v2.0. Nat. Protocols, v. 6, n. 9, p. 12901307, 2011. ISSN 1754-2189. Disponível em: <http://dx.doi.org/10.1038/nprot.2011.308>.

STEPHANOPOULOS, G.; ARISTIDOU, A. A.; NIELSEN, J. Metabolic engineering : principles and methodologies. San Diego ; London: Academic Press, 1998. xxi,725p. ISBN 0126662606.

TANGNEY, M.; MITCHELL, W. J. Analysis of a catabolic operon for sucrose transport and metabolism in Clostridium acetobutylicum ATCC 824. J Mol Microbiol Biotechnol, v. 2, n. 1, p. 71-80, Jan 2000. ISSN 1464-1801 (Print)
1464-1801
(Linking).
Disponível
em:

<http://www.ncbi.nlm.nih.gov/pubmed/10937490>.

TANGNEY, M.; WINTERS, G. T.; MITCHELL, W. J. Characterization of a maltose transport system in Clostridium acetobutylicum ATCC 824. J Ind Microbiol Biotechnol, v. 27, n. 5, p. 298-306, Nov 2001. ISSN 1367-5435 (Print)
$1367-5435$
(Linking).
Disponível
em:

<http://www.ncbi.nlm.nih.gov/pubmed/11781805>.

VASCONCELOS, I.; GIRBAL, L.; SOUCAILLE, P. Regulation of carbon and electron flow in Clostridium acetobutylicum grown in chemostat culture at neutral $\mathrm{pH}$ on mixtures of glucose and glycerol. Journal of Bacteriology, v. 176, n. 5, p. 1443-50, Mar 1994. ISSN 0021-9193 (Print)

0021-9193 (Linking). Disponível em:

<http://www.ncbi.nlm.nih.gov/pubmed/8113186>.

YU, Y. et al. Analysis of the mechanism and regulation of lactose transport and metabolism in Clostridium acetobutylicum ATCC 824. Appl Environ Microbiol, v. 73, n. 6, p. 1842-50, Mar 2007. ISSN 0099-2240 (Print)

0099-2240 (Linking). Disponível em:

<http://www.ncbi.nlm.nih.gov/pubmed/17209069>. 